\title{
Commentary on Associations of Radiographic Small Vessel Disease with Acute Intracerebral Hemorrhage Volume, Hematoma Expansion, and Intraventricular Hemorrhage
}

\author{
Sarah E. Nelson * (1)
}

@ 2019 Springer Science+Business Media, LLC, part of Springer Nature and Neurocritical Care Society

Venema et al. [1] performed a study based on Ethnic and Racial Variations in Intracerebral Hemorrhage, a multicenter, racially balanced, prospective study of spontaneous intracerebral hemorrhage (ICH). The authors hypothesize that underlying cerebral small vessel disease (CSVD) and poor outcome from $\mathrm{ICH}$ stem from two mechanisms: (1) how CSVD affects ICH severity, and (2) CSVD's influence on brain recovery after ICH; the authors intend to address the former in this study.

A total of $2579 \mathrm{ICH}$ patients (38\% of whom also had intraventricular hemorrhage [IVH]) were included in the study; they had a mean age of 61.7 years and median ICH volume of $10.5 \mathrm{~mL}$. The first non-contrast computed tomography (CT) scan on admission was evaluated, with available follow-up CTs evaluated for hematoma expansion. Patients with primary IVH, multiple ICH, poor imaging quality, and severe abnormalities (e.g., marked hydrocephalus, edema, and midline shift) were excluded. Extent of leukoaraiosis and cerebral atrophy, used in this study as markers of CSVD, were assessed using visual rating scales and were correlated with $\mathrm{ICH}$ volume, hematoma expansion, and IVH presence and severity (via the Graeb score) using univariate analyses and multivariable models. Associations between CSVD markers and $\mathrm{ICH}$ volume and hematoma expansion

*Correspondence: snelso43@jhmi.edu

Johns Hopkins University, Baltimore, MD, USA

This comment refers to the article available at https://doi.org/10.1007/ s12028-019-00876-4. were also stratified by hemorrhage location (lobar vs non-lobar).

The authors found that inter-rater reliability was good for all CSVD measurements. Increasing grades of atrophy were associated with lower ICH volume in a dosedependent manner after adjusting for confounders, while higher leukoaraiosis grades were associated with smaller ICH volumes in a dose-dependent way though this relationship was not statistically significant after Bonferroni correction. When stratifying by ICH location, atrophy was associated with decreased $\mathrm{ICH}$ volume in non-lobar $\mathrm{ICH}$; only grade 3 atrophy was associated with lower $\mathrm{ICH}$ volume for lobar $\mathrm{ICH}$. There was no association between $\mathrm{ICH}$ volume and leukoaraiosis for both lobar and nonlobar ICH. In addition, neither leukoaraiosis nor atrophy was associated with hematoma expansion. Increasing leukoaraiosis was associated with IVH occurrence, while atrophy was inversely associated with this outcome.

The study is well written, and the methods including statistics are extensive and thorough. The figures and tables (including supplementary tables) provide a clear summary of the relationship between the chosen CSVD markers and ICH and IVH variables. One primary finding was that their chosen markers of CSVD seemed to be associated with ICH (in particular IVH occurrence) in opposing ways. To explain this result, the authors hypothesize that the differing effects of atrophy and leukoaraiosis on IVH occurrence may be due to factors influencing these two markers that have not yet been fully elucidated. In addition, Venema et al's results suggest that CSVD and its association with poor outcome are not related to acute $\mathrm{ICH}$ presentation; this is because 
of the relationships found between the two CSVD markers and ICH and IVH variables: differing with regard to IVH presence, no relationship with respect to hematoma volume, and an association with smaller $\mathrm{ICH}$ volume for both markers.

Strengths of the paper include the large number of patients with $\mathrm{CT}$ available for review, validated scales used for assessing CSVD markers and ICH/IVH variables, and good inter-rater agreement. However, it should be noted that because patients with high-grade ICHs were less likely to be included, the results of this study may not be generalizable to these patients. Further, underlying CSVD pathology is complex and so these CTbased markers may not fully reflect CSVD burden; it is possible that magnetic resonance imaging (MRI) might have been helpful, but studies have shown agreement between MRI and CT visual rating scales for the chosen CSVD markers [2,3] and CT is more routinely available. In addition, the study contains no outcome data.

Venema et al's findings have potential implications for patients with leukoaraiosis and atrophy, especially in those at increased risk for ICH and IVH due to other factors. For instance, their results could potentially be used to predict how patients with varying degrees of atrophy and leukoaraiosis will fair in the event of ICH and/or IVH or to counsel patients regarding the risks and benefits of antithrombotic therapy.

Despite interesting results, this study's findings require further validation by including patients with a wide range of $\mathrm{ICH}$ volumes and severities. In addition, the use of MRI might be helpful to further tease out the relationships evaluated in this paper. Obtaining cognitive and functional outcome data would help shed additional light on the relationship between CSVD and ICH outcome including addressing the second mechanism for CSVD and poor outcome the authors discuss: CSVD's impact on brain recovery after $\mathrm{ICH}$. In addition, in order to use this study's methodology, the rating scales would need to be easily applied in the clinical setting.

\section{Publisher's Note}

Springer Nature remains neutral with regard to jurisdictional claims in published maps and institutional affiliations.

Published online: 3 December 2019

References

1. Venema SU, Marini S, Brouwers HB, Morotti A, Woo D, Anderson CD, Rosand J. Associations of radiographic cerebral small vessel disease with acute intracerebral hemorrhage volume, hematoma expansion, and intraventricular hemorrhage. Neurocrit Care. 2019. https://doi. org/10.1007/s12028-019-00876-4.

2. Wattjes MP, Henneman WJP, van der Flier WM, et al. Diagnostic imaging of patients in a memory clinic: comparison of MR imaging and 64-detector row CT. Radiology. 2009;253:174-83.

3. Ferguson KJ, Cvoro V, MacLullich AMJ, et al. Visual rating scales of white matter hyperintensities and atrophy: comparison of computed tomography and magnetic resonance imaging. J Stroke Cerebrovasc Dis. 2018;27:1815-21. 